\title{
Biomasses et croissance du pin maritime. Élude de la variabilité dans un peuplement de 16 ans *
}

\author{
B. LEMOINE *;, J. GELPE $* *$, J. RANGER $* * *$ et C. NYS $* * ;$ \\ * I.N.R.A., Laboratoire de Sylviculture et d'Ecologie \\ Domaine de l'Hermitage, Pierroton, F 33610 Cestas \\ *:* I.N.R.A., Station de Recherches sur les Sols forestiers et la Fortilisation \\ Centre de Recherches forestières de Nancy, Champenoux, F 54280 Seichamps
}

\section{Résumé}

Ce travail s'intègre dans une étude générale de l'écosystème forestier landais visant à juger l'effet de la sylviculture actuelle sur l'évolution de la fertilité minérale de l'écosystème (tendance à la récolte totale, fertilisation).

L'approche globale utilisée est celle du cycle biogéochimique. Elle nécessite l'évaluation des stocks (biomasse, minéralomasse, réserves du sol) et des principaux flux (prélèvements, retours).

Nous présentons les résultats concernant la morphologie et la biomasse d'un jeune peuplement (16 ans) de lande humide issu d'une expérimentation représentative de la sylviculture actuelle.

L'échantillonnage est effectué suivant la méthode stratifiée par classe de circonférence : 20 arbres sont retenus, un échantillonnage important est effectué au niveau du houppier (375 branches).

Une modélisation en cascade est nécessaire pour déterminer la biomasse du peuplement :

1) La modélisation de la biomasse des branches est établie à partir d'un modèle logarithmique prenant en compte le diamètre de la branche et son âge. Les écarts aux mesures montrent que ce modèle, bien que très satisfaisant, ne prend pas en compte l'effet « arbre». Notre échantillon ne permet pas de l'inclure dans le modèle.

2) La modélisation de la biomasse totale du houppier établie également à l'aide d'un modèle logarithmique montre que deux types de variables doivent être prise en compte : des variables contemporaines dı compartiment en question mais aussi des variables de croissance initiale de l'arbre représentant un caractère intrinsèque.

3) La biomasse compartimentée du peuplement issue de l'inventaire est déterminée à partir d'un modèle nécessairement simplifié ne faisant intervenir que des paramètres faciles à mesurer sur une surface représentative.

Mots clés : Biomasse, croissance, modélisation, Pinus pinaster (Ait.).

\footnotetext{
* Ce travail a fait lobjet d'une communication au Colloque I.U.F.R.O. « Mesures des biomasses et accroissements forestiers » qui s’est tenu à Orléans du 3 au 7 octobre 1983.
} 


\section{Introduction}

La forêt de pin maritime des Landes de Gascogne de création récente (150 ans) a fonctionné sur un million d'hectares jusqu'à une période proche de façon assez économe, grâce à des exportations généralement limitées aux bois écorcés.

L'intensification de la sylviculture a été très nette ces dernières années à cause de conditions techniques favorables : terrain plat, facile à travailler en raison de sa texture sableuse, mais aussi des résultats spectaculaires de recherches en matière de fertilisation phosphatée. Elle a débouché sur une augmentation importante de la production, chiffrée (en volume) par l'inventaire Forestier National pour la période 1961 à 1977 à +38 p. 100 dans le département de la Gironde et + 55 p. 100 dans le département des Landes. D'ici 30 ans, laccroissement moyen maximum du pin maritime de cette région atteindra environ la moitié de celui du Douglas en France.

Une étude globale de cet écosystème profondément modifié par l'homme a été entreprise. Elle vise à juger l'économie de la fertilisation minérale pratiquée ici, mais aussi l'influence des sollicitations nombreuses dont est l'objet cet écosystème (tendance à la récolte totale de la production) sur l'équilibre de la fertilité du sol. La compréhension du cycle biogéochimique revêt toute son importance sur les sols très pauvres de cette région.

Ce travail présente les premiers résultats intéressant la morphologie et la biomasse d'un jeune peuplement situé en lande humide. Le taux d'échantillonnage adopté dans ce travail permettra de plus une étude méthodologique, visant à optimiser réchantillonnage dans les prélèvements futurs.

\section{Matériel et méthodes}

Cette étude utilise une expérience de fertilisation situce sur un sol sableux (Landes de Gascogne) de type podzol humique sur lande humide à Molinie (Molinia coerulea M.). On n'a considéré ici qu'une des modalités de ce dispositif expérimental; le peuplement a été obtenu par semis, en juillet 1963, sur bandes labourées de 2 mètres, distantes de 3 mètres; on a apporté initialement 100 unités/ha de $P_{2 .} O_{z}$, sur la bande (scories), ce qui correspond à la pratique courante ; on a procédé à un renouvellement de dose 10 ans après, soit 80 unités/ha.

Afin que le caractère destructif de l'étude ne nuise pals à la survie de lexpérimentation, on a effectué les prélèvements d’arbres sur deux placeaux appartenant au même traitement, mais appartenant à deux blocs de vigueurs voisines.

Ces deux placeaux, chacun d'une superficie de 12,60 ares, avaient, à 16 ans, les caractéristiques dendrométriques figurant dans le tableau récapitulatif 4.

Ces deux placeaux se rattachent à la Classe 1 de fertilité de la Table de Production (Decourt N., Lemoine B., 1969); le deuxième est légèrement plus fertile d'une demi-classe, et plus dense, que le premier. Par contre, les circonférences moyennes sont pratiquement identiques. 
On a considéré que ces deux placeaux représentaient le même peuplement. On a alors procédé à un échantillonnage, d'arbres, stratifié à partir de l'inventaire des circonférences à $1,30 \mathrm{~m}$. On a ainsi retenu 10 arbres dans chacun des deux placeaux.

Sur chacun des 20 arbres abattus on a procédé de la façon suivante :

- au niveau du houppier : afin de limiter le nombre de déterminations de matières sèches on a procédé au sein de chaque arbre à un échantillonnage de branches et à la construction de tarifs; tout en faisant l'inventaire en diamètre de toutes les branches - diamètre basal à $10 \mathrm{~cm}$ de l'insertion $=\mathrm{D} 10-$ on a adopté pour celles-ci, tout le long de la couronne, un taux d'échantillonnage de $1 / 3$; on s'est efforcé de se rapprocher d'un échantillonnage stratifié en retenant, pour chaque âge ou verticille, la branche la plus grosse et la plus petite (d'un diamètre basal toutefois supérieur à $5 \mathrm{~mm}$ ) et parfois la branche moyenne : cette modalité pratique s'est révélée être tout à la fois la plus rapide et la plus facile à mettre en cuvre. On a ensuite déterminé les matières sèches, foliaire et ligneuse, de ces branches échantillons (soit 375 branches sur 1012 branches inventoriées);

- au niveau du tronc: on a débité et pesé des billons d'un mètre, puis prélevé des rondelles à leurs extrémités afin, d'une part de déterminer les densités infraspécifiques et les parts respectives, en matière sèche, du bois et de l'écorce, d'autre part de caractériser l'historique de la croissance (par analyse de tige);

- au niveau des racines : on a extrait, au moyen d'un tire-fort, les souches de 18 des 20 arbres; après la pesée de chacune d'entre elles on y a prélevé des échantillons pour en déterminer la densité infraspécifique.

Les prélèvements ont été effectués en mars 1979.

L'interprétation des données mesurées fait appel aux outils statistiques suivants :

- l'analyse de régression multiple, descendante :

- l'analyse de covariance multiple : les covariables finalement retenues sont déterminées comme les variables explicatives de la régression multiple descendante ; cette analyse est utilisée uniquement pour la construction des tarifs biomasses ligneuse et foliaire des branches à l'intérieur des houppiers;

- la construction de matrices de corrélations et, éventuellement, leur interprétation par l'analyse en composantes principales.

\section{Résultats}

\subsection{Résultats intermédiaires}

\subsection{Estimation des biomasses des branches vivantes}

On rappellera au préalable deux caractéristiques importantes du pin maritime :

- la durée de vie moyenne de ses aiguilles est très peu supérieure à 2 ans ;

- ses populations comprennent des arbres soit polycycliques ( 2 pousses par an), soit monocycliques (une pousse par an), soit encore intermédiaircs ( 1 ou 2 pousses selon les années); sur 20 arbres nous avons relevé pour ces trois types les effectifs 
respectifs suivants : 3,14 et 3 ; ces nombres sont, compte tenu de notre échantillonnage peu différents de ceux obtenus par KrEMER, 1981. Dans la réalisation des calculs, les branches issues des premières et des secondes pousses annuelles ont été regroupées.

On cherche, à partir de l'échantillon de branches, à construire des tarifs destinés à estimer les biomasses des houppiers des 20 arbres. Dans les régressions calculées, les variables à expliquer sont la biomasse ligneuse, MS b, et foliaire MS $f$ en $g$, de chaque branche. On utilise comme variable explicatrice le diamètre mesuré à la base de la branche, D $10 \mathrm{cn} \mathrm{mm}$. On a alors les modèles suivants :

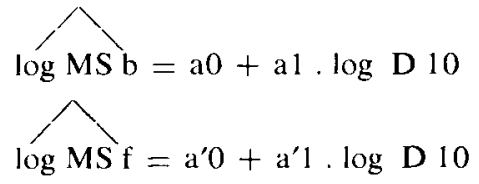

On sait déjà que les coefficients de ces tarifs varient selon le niveau dans la couronne (Ranger et al., 1981), d'où l'idée de considérer comme variable explicatrice supplémentaire la hauteur relative, ou hauteur d'insertion rapportée à la hauteur totale de l'arbre : MADGWICK \& JACKSON (1974) trouvent que l'effet de cette variable sur les biomasses est du type parabolique; de plus aux effets des variables diamètre et hauteur relative se superposerait un effet clone (génotype). On préfère utiliser ici comme variable explicative supplémentaire l'âge de la branche, $A$. facile à déterminer dans le cas du pin maritime. On a alors les modèles suivants :

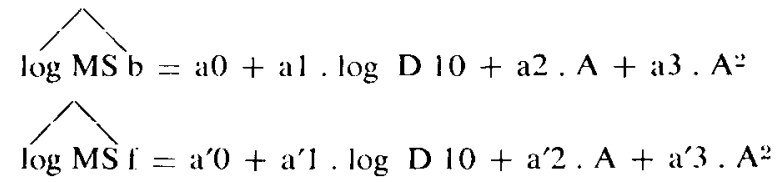

On recherche ici l'éventualité d’un effet arbre (phénotype). L'analyse de covariance fournit les résultats suivants détaillés dans le tableau 1 :

- Papport des covariables $A$ et $A^{2}$ est significatif, aussi bien pour l'explication de la biomasse foliaire - équation (2') - que pour celle de la biomasse ligneuse équation (2) ;

- les 20 plans de régression (dans l'espace à 4 dimensions) ayant trait à chaque arbre diffèrent les uns des autres : dans le cas des biomasses foliaires ils ne sont pas parallèles; dans le cas des biomasses ligneuses ils sont parallèles mais non confondus.

Le tableau 2 présente chacun des 20 couples d'équations (2) et (2') propre à chacun des 20 arbres ainsi que le couple des équations dites régressions intracommunes; ces deux dernières nous renseignent sur les caractéristiques moyennes du modèle mais ne peuvent être utilisées sans risquer une surestimation ou une sousestimation des biomasses de certains arbres. L'analyse de covariance montre qu'aux effets sur les biomasses des variables diamètre et âge de la branche se superpose un effet arbre sur les variations des coefficients de régression (biomasses foliaires) ou sur la variation de la constante (moyennes ajustées de la biomasse ligneuse).

On a tenté d'interpréter ces variations :

- en ce qui concerne la biomasse ligneuse : on a trouvé (Lemolne ét al., 1983) que la longueur $L$ de la branche était une fonction de son diamètre $D 10$, de son âge A et de l'arbre. Cet effet arbre (valeur de la constante de l'équation de régression multiple, estimant la longueur) explique 51 p. 100 de la variation de la constante 


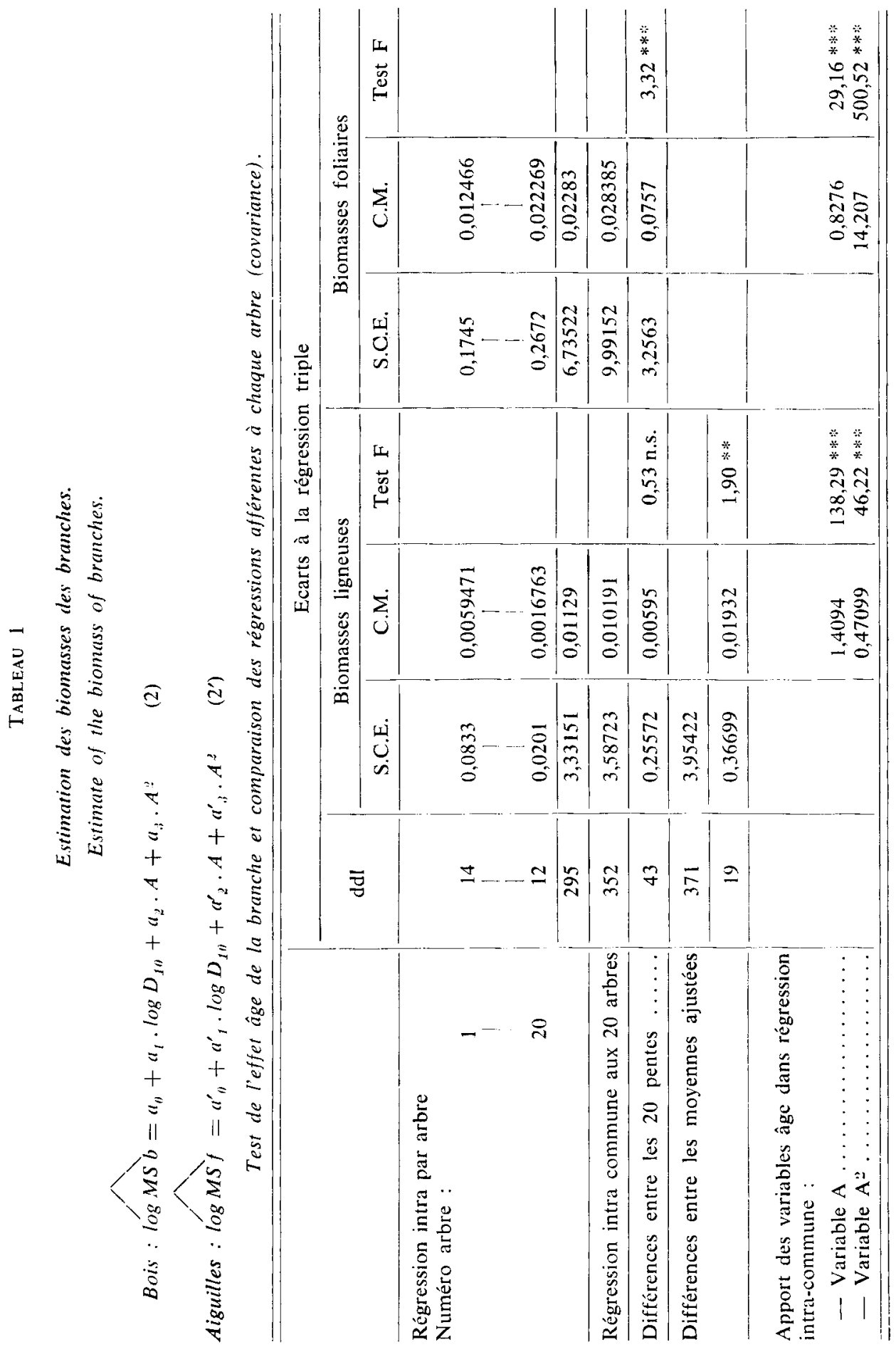




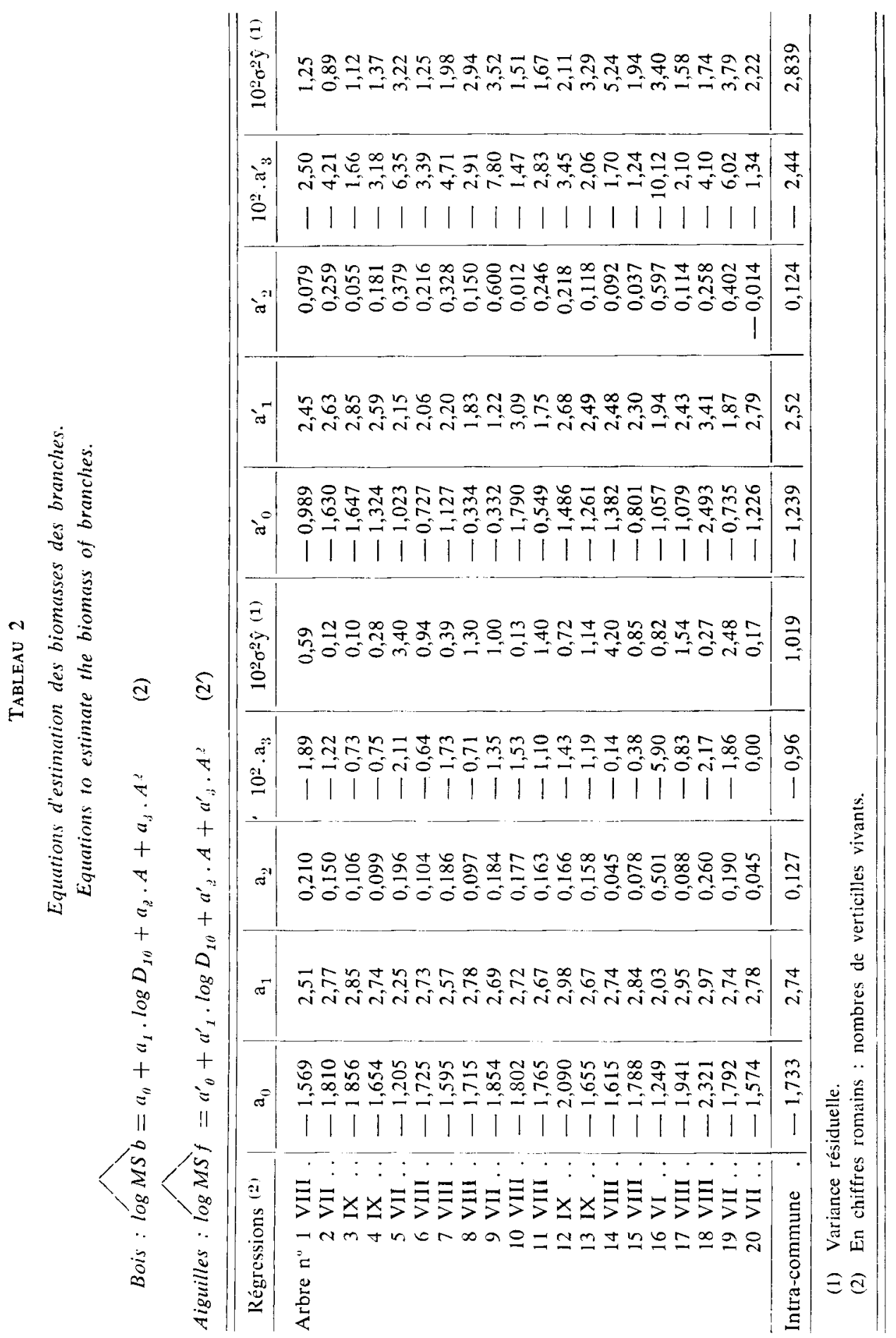


a0 du tarif. Un tarif à trois entrées - D 10, A, L - devrait donc être plus stable, d'un arbre à l'autre, qu'un tarif à deux entrées - D 10, A - ;

- en ce qui concerne la biomasse foliaire : les coefficients de l'équation (2') varient semble-t-il en fonction du nombre de verticilles vivants de l'arbre (cf. tableau 2) ; quand ce nombre augmente l'effet de l'âge de la branche dans l'arbre parait diminuer (valeurs de $a^{\prime} 2$ et de $a^{\prime} 3$ plus faibles) et l'effet du diamètre augmenter (valeurs de a'1 plus fortes). Les valeurs des coefficients de l'équation (2) suivent d'ailleurs ceux de l'équation $\left(2^{\prime}\right)$. Cette tendance sera à vérifier à l'aide d'autres échantillons d'arbres.

On considère maintenant en particulier une quelconque des 20 équations (2) ou (2). L'antilogarithme de la variable expliquée fournit une estimation biaisée des biomasses. BASKERville (1972) à la suite d'autres auteurs, expose le calcul permettant de corriger ce biais : la meilleure estimation de nos biomasses sera alors :

MS $b=10^{\log M S b} \cdot 10^{2 / 2}$

ou

$\operatorname{MS} \mathrm{f}=10^{\log \mathrm{MS} \mathrm{f}} \cdot 10^{0 \mathrm{\alpha} / 2}$

où $\widehat{\sigma^{2}}=$ variance résiduelle de la régression $(2)$ ou $\left(2^{\prime}\right)$; les quantités log MS b ou $\log$ MS f d'une part, et MS b ou MS f d'autre part, sont de natures différentes : les premières sont des estimations issues de l'emploi des équations (2) et (2'), les secondes des valeurs plus proches de la réalité que l'antilogarithme des premières.

Le facteur de correction $10 \widehat{0} 2 / 2$ change d'un arbre à l'autre et d'un compartiment à l'autre (cf. tabl. 1), citons les extrêmes suivants :

$\begin{array}{ccc} & \text { arbre } 2 & \text { arbre } 14 \\ \text { MS b } & 1,001 & 1,050 \\ \text { MS f } & 1,010 & 1,062\end{array}$

3.12. Profils de répartition verticale des biomasses estimées du houppier; branches vivantes

On regroupe les vingt arbres en trois types selon le nombre de verticilles vivants observés qui varie de neuf à six. La figure 1 présente le profil moyen de trois de ces types (le type à six verticilles ne concerne qu'un seul arbre) et le tableau ci-dessous les moyennes par type d'arbre de la biomasse foliaire $2 \mathrm{MS} \mathrm{f}$, de la biomasse ligneuse totale des branches $\Sigma$ MS b et de la hauteur totale $h$

\begin{tabular}{|c|c|c|c|}
\hline arbres à : & ${\overline{\Sigma M S f_{l k m}}}$ & $\overline{\Sigma M S ~}_{\mathrm{lig}}$ & $\mathbf{h}_{\mathrm{n} 1}$ \\
\hline 9 verticilles & 11,8 & 22,6 & 12,2 \\
\hline 8 verticilles & 9,2 & 14,4 & 12,0 \\
\hline 7 verticilles & 8,4 & 9,2 & 11,6 \\
\hline
\end{tabular}

On peut faire les remarques suivantes : du haut ;

- les profils ne se différencient nettement qu'à partir du 4" verticille en partant

- le type à neuf verticilles se distingue des types à huit et sept verticilles par une augmentation importante de la biomasse ligneuse totale des branches; 


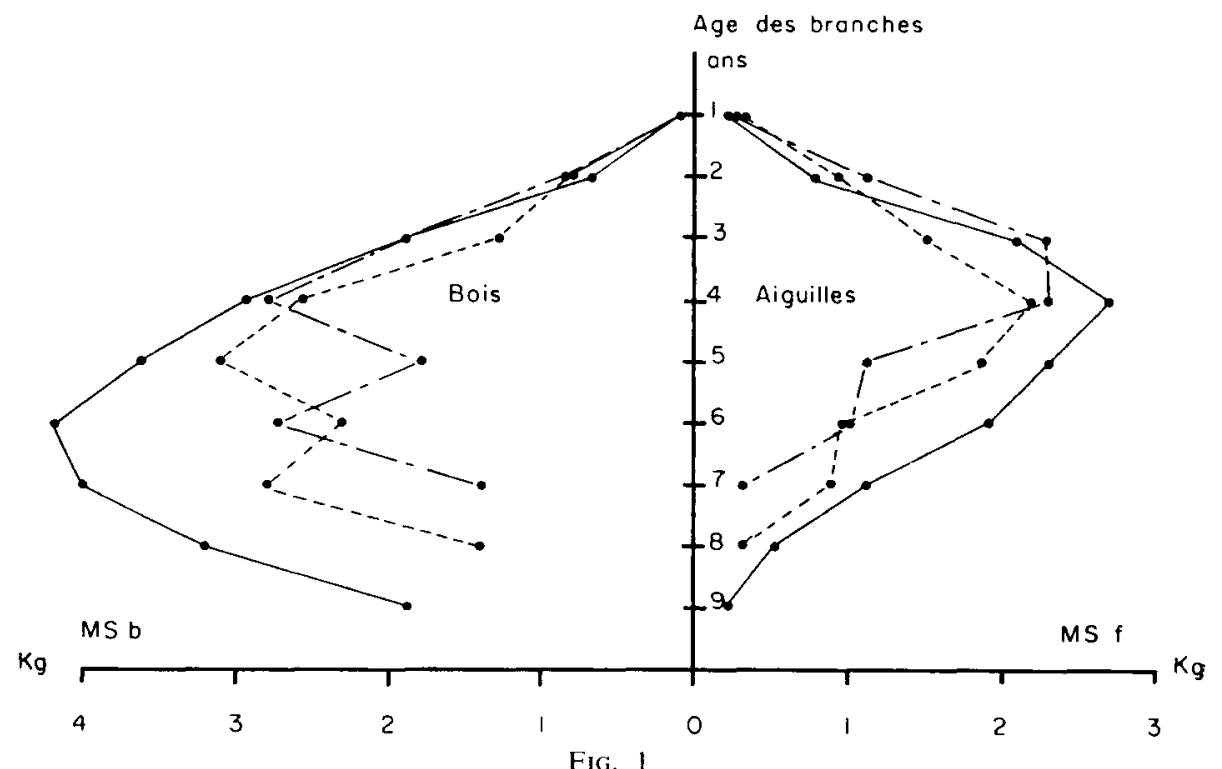

FIG. 1

Profils moyens de répartition verticale des biomasses estimées du houppier. Mean profiles for a vertical distribution of estimated biomass for the crown.

Branches vivantes - Living branches :

4 arbres à 9 verticilles vivants.

4 trees with 9 living whorls.

10 arbres à 8 verticilles vivants.

10 trees with 8 living whorls.

- - . 5 arbres à 7 verticilles vivants.

5 trees with 7 living whorls. branches ;

- la biomasse foliaire totale varie moins que la biomasse ligneuse totale des

- la hauteur de l'arbre varie très faiblement d'un type à lautre (la variation à l'intérieur de chaque type est plus importante).

\subsection{Croissance du tronc, en section}

On a choisi d'appréhender la croissance du tronc par les accroissements en surface des cernes Ig plutôt que par leurs accroissements radiaux. Les premiers sont en effet plus représentatifs des accroissements en volume du tronc que les seconds.

Sur les rondelles les plus basses de chacun des 20 arbres situćes à $0,50 \mathrm{~m}$ audessus du sol, on dispose de 14 accroissements successifs en surface de cerne. On forme ainsi un tableau de données à 14 variables et 20 individus, que l'on traite par une analyse factorielle en composantes principales.

Les 14 variables Igj ( $\mathrm{j}=$ âge) donnent naissance à 2 composantes $\mathrm{Y} 1$ et $\mathrm{Y} 2$, qui absorbent respectivement 68,52 p. 100 et 21,10 p. 100 de la variabilité. La figure 2 situe les extrémités des quatorze vecteurs représentatifs des variables initiales sur le plan de référence déterminé par ces 2 composantes. 


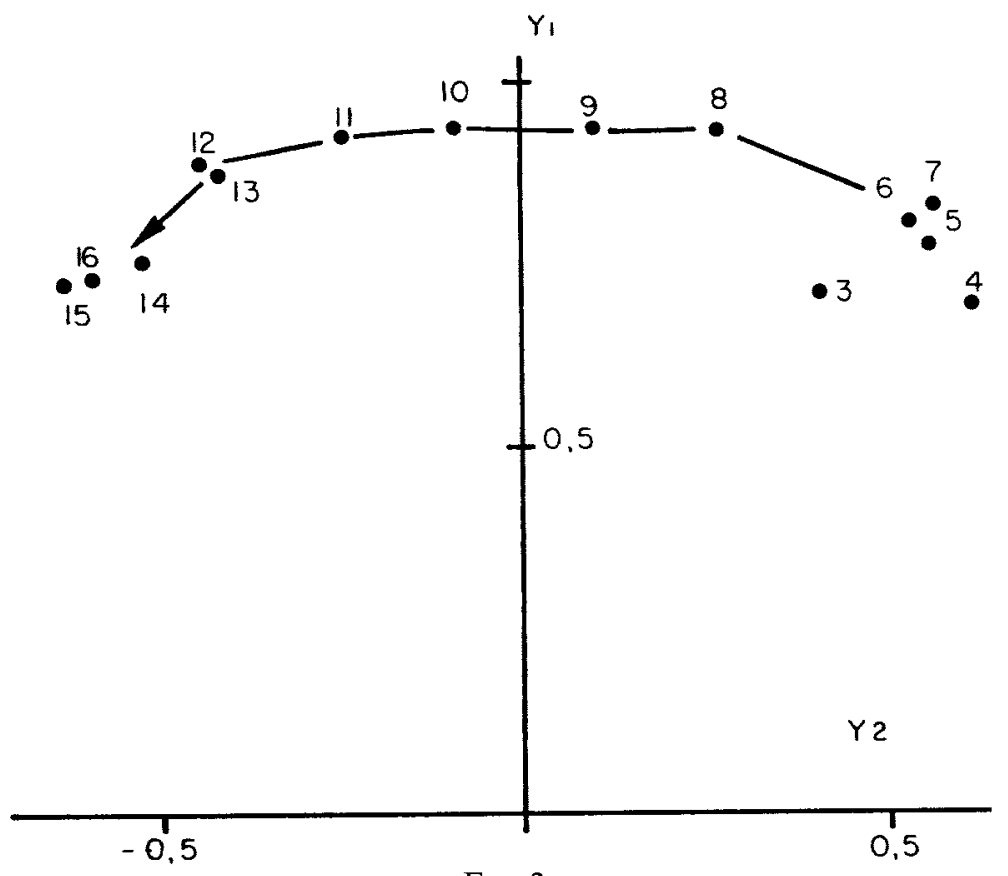

FIG. 2

Croissance en surface des cernes $d u$ bas du tronc. Analyse en composantes principales.

Growth of the surface area annual rings at the base of the trank. Principal components analysis.

Trace des variables Igj: accroissements annuels sur le plan des 2 composantes.

Graph of the variable Igj : annual growth on the plan of the 2 components.

Numéros : âge.

Number : age.

Une structure mathématique analogue à celle que nous mettons en évidence ici peut se rencontrer dans d'autres domaines de la biologie. Se basant sur la même interprétation formelle et utilisant la même terminologie que celles de Buss (1967) on peut dire qu'on a affaire à une "configuration de croissance »:

— les «extrémités des vecteurs-tailles », c'est-à-dire des vecteurs représentatifs des variables initiales dans l'espace de référence (ici un plan), voient «leurs extrémités situées dans l'ordre même de la succession chronologique des temps qu'ils représentent... $\gg ;$

— «les vectcurs-tailles sont groupés d'une certaine manière ».

Nous observons dans notre cas :

— un stade initial avec le groupe des variables $\lg 3$ à $\operatorname{Ig} 7$, très corrélées entre elles ;

— un stade final avec le groupe des variables $\operatorname{Ig} 14$ à $\operatorname{Ig} 16$, très corrélées entre elles. 
Ces deux groupes de variables sont pratiquement orthogonaux (non corrélés entre eux). On observe également que six variables font transition entre ces deux groupes.

On peut tenter d'interpréter cettc description mathématique de la croissance en faisant référence à des travaux antérieurs sur la concurrence (ILLY \& LEMOINE, 1970; Lemoine, 1980); la figure 3 fait état :

- de l'évolution théorique, selon l'âge du peuplement, des deux espacements minimas entre les arbres pour que ceux-ci restent exempts, soit de toute concurrence, même dans le sol (courbe supérieure), soit d'une très forte concurrence, à la fois pour la lumière et dans le sol (courbe inférieure):

- des espacements moyens, estimés, à la suite de comptages, sur la bande labourée lors des dépressages et éclaircies faits par le sylviculteur dans les placeaux étudiés ici.

espocement moyen entre les arbres

$\mathrm{m}$.

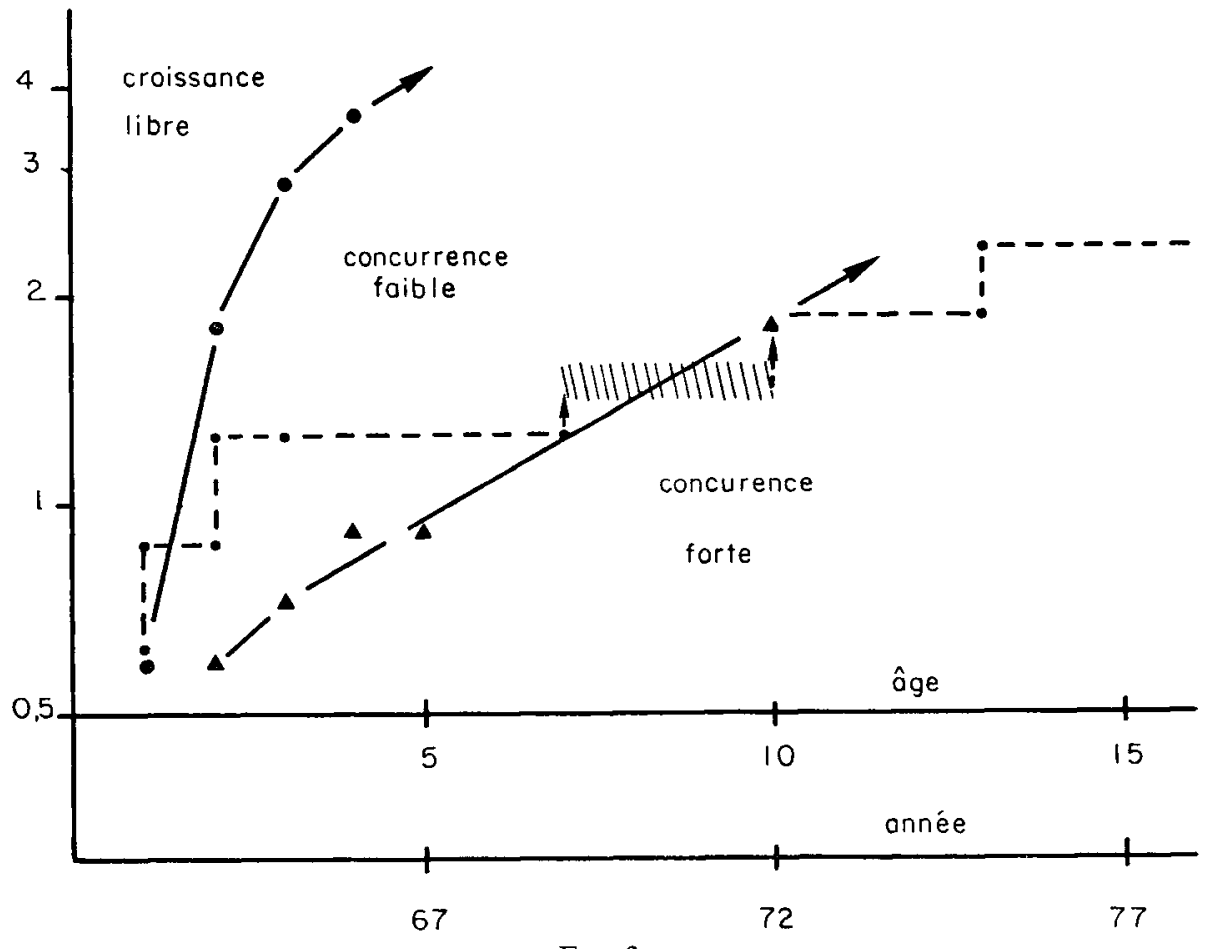

FIG. 3

Evolution théorique du statut de concurrence du peuplement.

Theoretical evolution of the laws of competition in the stand.

Courbes en traits pleins : modèle expérimental sur la concurrence, espacement entre arbres en tous sens.

Ligne brisée en tiretés : peuplement étudié, espacement moyen entre les arbres sur la bande labourée.

Unbroken lines : experimental model of the competition, spacing between trees in all directions.

Jagged broken lines: stand studied, mean spacing between trees in the ploughed banas. 
On observe que l'on quitte le stade initial pour évoluer vers le stade final (fig. 2) en 1970, au moment où le sylviculteur n'élimine plus systématiquement la concurrence pour la lumière, c'est-à-dire à l'âge de 8 ans (fig. 3). Cette concurrence pourrait donc être responsable du bouleversement complet du classement des accroissements individuels établi dans les premières années.

On devra vérifier cette hypothèse sur nos dispositifs d'étude de la concurrence : à une densité de plantation plus ou moins forte correspond une fermeture de couvert plus ou moins précoce qui devrait entraîner un bouleversement également plus précoce du classement des accroissements individuels établi dans les premières années.

Si cette hypothèse de «configuration de croissance» était vérifiée, on pourrait en tirer des considérations sylvicoles : un peuplement du même type que celui-ci est constitué d'arbres dont quelques-uns seulement ont une production à la fois forte et soutenue ; il serait alors de la plus haute utilité de rechercher un modèle de dépressage et d'éclaircic permettant de maximiser ce nombre d'arbres à «croissance rapide $\gg$.

Pour la suite de cette étude il convient donc d'utiliser suffisamment de variables représentatives de la configuration de croissance : nous avons retenu les accroissements $\operatorname{Ig} 7, \operatorname{Ig} 8$, $\operatorname{Ig} 10, \operatorname{Ig} 13$ et $\operatorname{Ig} 16$.

\subsection{Résultats globaux}

La première partie de cette étude est prospective : on se met dans la situation où l'on aurait auparavant mesuré, sur tous les arbres du peuplement, plusieurs variables explicatives des biomasses du houppier afin de calculer des tarifs à plusieurs entrées par régression multiple.

La deuxième partie a trait à la construction et à l'application de tarifs à une seule entrée, la surface terrière de l'arbre, de manière à évaluer chacun des compartiments de biomasses du peuplement étudié.

\subsection{Recherches prospectives concernant la variabilité et l'estimation des biomasses des branches du houppier}

On citera au préalable quelques travaux antérieurs :

- Kittredge (1944) formule la remarque suivante : «sur le plan théorique on peut s'attendre à ce que la quantité de feuillage puisse être estimée à partir de la croissance annuelle périodique parce que l'accroissement en bois du tronc est déterminé par la quantité de feuillage qui exerce la photosynthèse pendant cette période $\gg$;

- Satoo \& MAdGWick (1982) trouvent que la variable supplémentaire longueur de cime améliore la prévision, faite par la circonférence à $1,30 \mathrm{~m}$ de la biomasse des branches du houppier;

- Plusieurs auteurs, dont Storey (in Satoo \& Madgwick, 1982) confirment que la circonférence du tronc à la base de la couronne vivante est un bon estimateur de la biomasse des branches du houppier. 


\section{TABLEAU 3}

Estimation des biomasses branches du houppier.

Régressions logarithmiques multiples descendantes.

Biomasses ligneuses branches $=\log \Sigma$ MS b.

Analyse de variance globale.

(Test de l'apport de chaque variable) après variable $X_{11}=\log c^{\prime}$.

\begin{tabular}{|c|c|c|c|c|}
\hline & Palier & Deg. liberté & Carré moyen & Test $\mathrm{F}$ \\
\hline Apport total $\ldots \ldots \ldots \ldots \ldots \ldots$ & 1 & 9 & $1,0740 \mathrm{E}-01$ & 61,03 \\
\hline Variable $X \quad 6=\log \operatorname{Ig} 8$ & 2 & $\begin{array}{l}8 \\
1\end{array}$ & $\begin{array}{l}1,2082 \mathrm{E}-01 \\
2,9605 \mathrm{E}-05\end{array}$ & $\begin{array}{r}68,65 \\
0,02\end{array}$ \\
\hline $\begin{array}{l}\text { Apport total } \ldots \ldots \ldots \ldots \ldots \ldots \ldots \\
\text { Variable } x 4=\log \lg 13 \ldots \ldots \ldots\end{array}$ & 3 & $\begin{array}{l}7 \\
1\end{array}$ & $\begin{array}{l}1,3792 \mathrm{E}-01 \\
1,0761 \mathrm{E}-03\end{array}$ & $\begin{array}{r}78,37 \\
0,61\end{array}$ \\
\hline $\begin{array}{l}\text { Apport total } \ldots \ldots \ldots \ldots \ldots \ldots \ldots \\
\text { Variable } X \quad 8=\log c \ldots \ldots \ldots \ldots\end{array}$ & 4 & $\begin{array}{l}6 \\
1\end{array}$ & $\begin{array}{l}1,6067 \mathrm{E}-01 \\
1,4490 \mathrm{E}-03\end{array}$ & $\begin{array}{r}91,30 \\
0,82\end{array}$ \\
\hline $\begin{array}{l}\text { Apport total } \cdots \cdots \cdots \\
\text { Variable } X \quad 5=\log \lg 10 \ldots \ldots \ldots\end{array}$ & 5 & $\begin{array}{l}5 \\
1\end{array}$ & $\begin{array}{l}1,9263 \mathrm{E}-01 \\
8,7374 \mathrm{E}-04\end{array}$ & $\begin{array}{r}109,46 \\
0,50\end{array}$ \\
\hline $\begin{array}{l}\text { Apport total } \ldots \ldots \ldots \ldots \ldots \ldots \\
\text { Variable } X \quad 9=\log h \ldots \ldots \ldots \ldots\end{array}$ & 6 & $\begin{array}{l}4 \\
1\end{array}$ & $\begin{array}{l}2,4027 \mathrm{E}-01 \\
2,0559 \mathrm{E}-03\end{array}$ & $\begin{array}{r}136,53 \\
1,17\end{array}$ \\
\hline $\begin{array}{l}\text { Apport total } \ldots \ldots \ldots \ldots \ldots \\
\text { Variable } x \quad 3=\log \lg 16 \ldots \ldots \ldots\end{array}$ & 7 & $\begin{array}{l}3 \\
1\end{array}$ & $\begin{array}{l}3,1662 \mathrm{E}-01 \\
1,1233 \mathrm{E}-02\end{array}$ & $\begin{array}{r}179,91 \\
6,38\end{array}$ \\
\hline $\begin{array}{l}\text { Apport total } \ldots \ldots \ldots \ldots \ldots \\
\text { Variable } \times 10=\log 1 \ldots \ldots \ldots\end{array}$ & 8 & $\begin{array}{l}2 \\
1\end{array}$ & $\begin{array}{l}4,6659 \mathrm{E}-01 \\
1,6670 \mathrm{E}-02\end{array}$ & $\begin{array}{r}265,14 \\
9,47\end{array}$ \\
\hline $\begin{array}{l}\text { Apport total } \cdots \cdots \cdots \cdots \\
\text { Variable } x \quad=\log \lg 7\end{array}$ & 9 & $\begin{array}{l}1 \\
1\end{array}$ & $\begin{array}{l}8,9946 \mathrm{E}-01 \\
3,3725 \mathrm{E}-02\end{array}$ & $\begin{array}{r}511,11 \\
19,16\end{array}$ \\
\hline Déviations & & 10 & $1,7598 \mathrm{E}-03$ & \\
\hline
\end{tabular}

Nous traitons alors nos données en mettant en concurrence toutes les variables explicatives disponibles dans notre échantillon de 20 arbres :

- les accroissements en section du tronc $\operatorname{Ig} 16, \operatorname{Ig} 13, \lg 10, \operatorname{Ig} 8$ et $\operatorname{Ig} 7$, sélectionnés par l'analyse ci-dessus (\$3.13), en $\mathrm{cm}^{2}$;

- la circonférence à $1,30 \mathrm{~m}, \mathrm{c}$ en $\mathrm{mm}$, et la hauteur totale, $\mathrm{h}$ en $\mathrm{cm}$, de l'arbre ;

- la circonférence du trone à la base de la couronne vivante, é en mm, et la longueur de cette couronne 1 en $\mathrm{cm}$.

Nous optons ici pour des régressions logarithmiques afin de ne pas avoir à faire à un modèle linéaire rendu trop lourd par l'adjonction de termes multiplicatifs (combinaisons de variables). 
Estimate of the biomass of the crown branches. Multiple forward selection regression after a logarithmic transformation of the variables.

Biomasses foliaires $=\log \Sigma M S f$. Analyse de variance globale. (Test de l'apport de chaque variable) après variable $X_{3}=\log I \quad g 16$.

\begin{tabular}{|c|c|c|c|c|}
\hline & Palier & Deg. liberté & Carré moyen & Test $\mathrm{F}$ \\
\hline Apport total ... & I & 9 & $4,7463 \mathrm{E}-02$ & 6,90 \\
\hline Apport total $\ldots \ldots \ldots$ & & 8 & $5,3396 \mathrm{E}-02$ & 7,77 \\
\hline Variable $\times \quad 7=\log \operatorname{Ig} 7$ & 2 & 1 & $3,3332 \mathrm{E}-06$ & 0,00 \\
\hline Apport total $\ldots \ldots \ldots \ldots$ & & 7 & $6,1023 \mathrm{E}-02$ & 8,88 \\
\hline Variable $\mathrm{X} 9 \log \mathrm{h} \ldots$ & 3 & 1 & $8,5612 \mathrm{E}-06$ & 0,00 \\
\hline Apport total $\ldots \ldots \ldots \ldots$ & & 6 & $7,1117 \mathrm{E}-02$ & 10,34 \\
\hline Variable $X 5=\log \operatorname{Ig} 10 \ldots$ & 4 & 1 & $4,5976 \mathrm{E}-04$ & 0,07 \\
\hline Apport total $\ldots \ldots \ldots \ldots \ldots \ldots$ & & 5 & $8,4038 \mathrm{E}-(02$ & 12,34 \\
\hline Variable $X \quad 8=\log c \ldots \ldots \ldots \ldots$ & 5 & 1 & $2,5111 \mathrm{E}-03$ & 0,37 \\
\hline Apport total $\ldots \ldots \ldots \ldots \ldots \ldots$ & & 4 & $1,0535 \mathrm{E}-01$ & 15,32 \\
\hline Variable $\times 11=\log c^{\prime} \ldots$ & 6 & 1 & $2,7714 \mathrm{E}-03$ & 0,40 \\
\hline Apport total $\ldots \ldots \ldots \ldots$ & & 3 & $1,3667 \mathrm{E}-01$ & 19,83 \\
\hline Variable $\times 4=\log \lg 13$ & 7 & 1 & $1,1417 \mathrm{E}-02$ & 1,66 \\
\hline Apport total $\ldots \ldots \ldots$ & & 2 & $1,9674 \mathrm{E}-01$ & 28,62 \\
\hline Variable $\times 10=\log 1$ & 8 & 1 & $1,6515 \mathrm{E}-02$ & 2,40 \\
\hline Apport total $\ldots \ldots \ldots \ldots$ & & 1 & $3,4308 \mathrm{E}-01$ & 49,90 \\
\hline Variable $X \quad 6=\log \operatorname{Ig} 8 \ldots \ldots \ldots$ & 9 & 1 & $5.0403 \mathrm{E}-02$ & 7,33 \\
\hline Déviations & & 10 & $6,8753 \mathrm{E}-03$ & \\
\hline
\end{tabular}

On peut retenir aux vues des analyses de variance présentées dans le tableau 3, les équations de régression suivantes qui rendent compte de la biomasse foliaire $\Sigma$ MS $\mathrm{f}$ et de la biomasse ligneuse, $\Sigma$ MS b, des branches du houppier, en grammes :

$\log \Sigma M S b=5,934+0,285 \cdot \log \operatorname{Ig} 16+0,352 \cdot \log \operatorname{Ig} 7+0,971 . \log 1$

$+1.347 . \log \mathrm{c}^{\prime}$

$R=0,9882(0,9394$ avec la seule variable explicatrice $\log c)$.

$\log \Sigma \mathrm{MSf}=0,154+0,520 \cdot \log \operatorname{Ig} 16+0,365 \cdot \log \operatorname{Ig} 8$

$\mathrm{R}=0,8907(0,8706$ avec la seule variable explicatrice $\log \mathrm{c})$. 
On voit que :

- la remarque de Kittredge concernant la masse foliaire et faisant référence à la photosynthèse est justifiée : de tous les coefficients de régression concernant les accroissements celui de Ig 16 dans l'équation (3') est le plus fort;

- la biomasse ligneuse des branches du houppier et sa biomasse foliaire ont chacune les deux mêmes "composantes », la croissance du premier stade et la croissance du dernier stade:

- la biomasse ligneuse branche est très corrélée avec les caractéristiques globales, lc et $c^{\prime}$ de la portion du tronc garnie de branches vivantes; on peut voir là une corrélation de croissance entre les deux biomasses du houppier, aux âges strictement identiques, celle des branches et celle appartenant à la tige ;

- les variances résiduelles sont respectivement de $1,76.10^{-3}$ pour la biomasse ligneuse et de $6,88 \cdot 10^{-3}$ pour la biomasse foliaire : certaines causes de variation de cette dernière n'ont donc pu être appréhendées.

Sur le plan pratique nous pensons :

- qu'il n'est pas opportun de modifier les modalités de l'échantillonnage d'arbres servant à l'estimation de la masse foliaire du peuplement ;

- qu'il est possible, en théorie, de retenir un échantillon d'arbres plus petit pour l'estimation de la biomasse ligneuse des branches vivantes; ceci permet de diminuer l'importance des séchages à l'étuve mais introduit une contrainte supplémentaire : on doit alors disposer pour tous les arbres du placeau étudié (ici une centaine) de quatre caractères dendrométriques; or les mesures d'accroissements pćriodiques ne sont disponibles qu'au sein de dispositifs expérimentaux ou de placettes semi-permanentes.
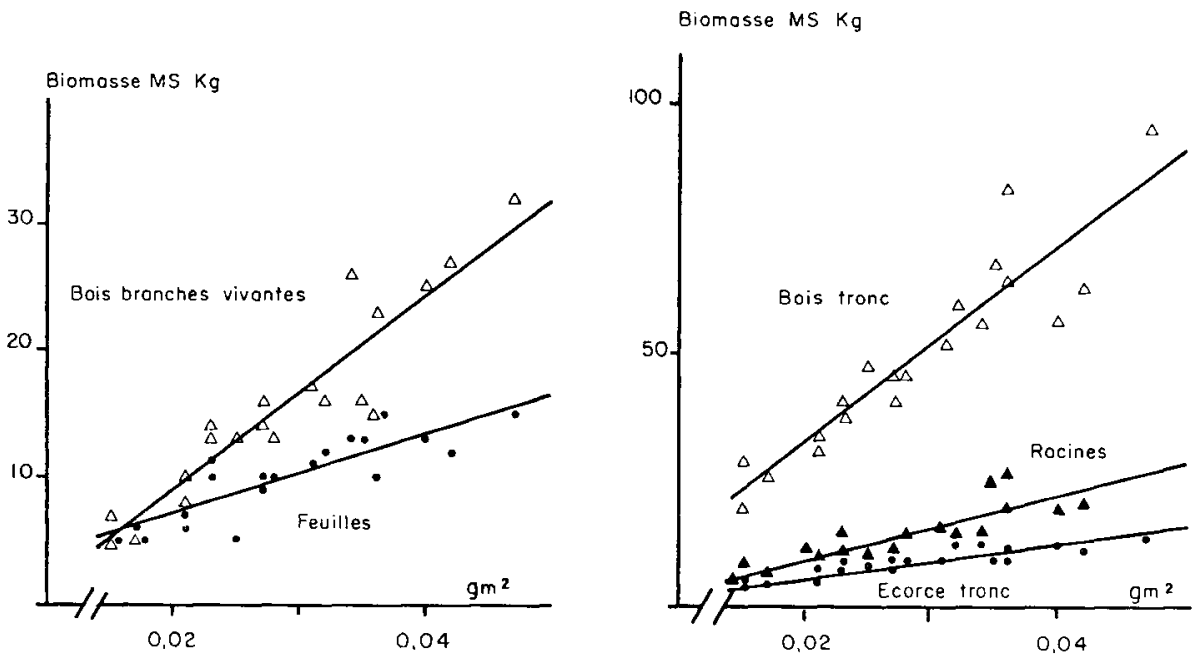

FIG. 4

Relation entre la biomasse de chacun des compartiments $C_{k}$ de l'arbre et de la surface terrière de celui-ci (équations 4).

The relationship between the biomass of each part of the tree $C_{k}$ and its basal area. 


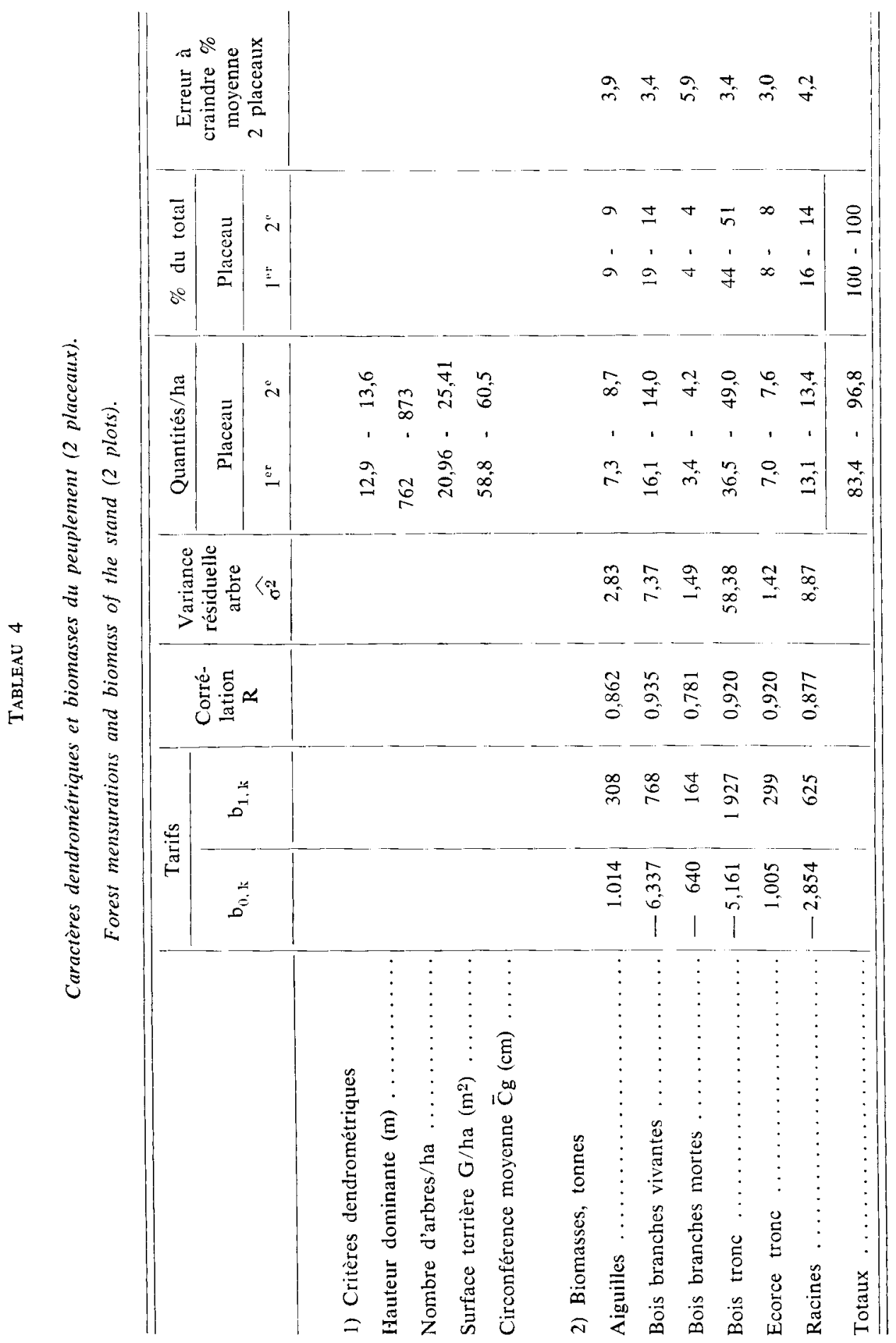




\subsection{Estimation des compartiments de biomasses du peuplement étudié}

Nous revenons ici à notre objectif principal qui est d'estimer les biomasses sur pied du peuplement étudié qui est représentatif de la sylviculture actuelle des Landes de Gascogne. On opte pour une approche statistique classique compatible avec le type de données recueillies.

On a individualisé six compartiments $\mathrm{Ck}$ : les aiguilles, le bois des branches vivantes et celui des branches mortes, le bois et l'écorce du tronc et la biomasse racinaire extraite.

Ces six compartiments font l'objet d'une estimation par l'intermédiaire d'un tarif à une entrée de la forme :

$$
\mathrm{Ck}=\mathrm{b}_{\mathrm{t} . \mathrm{k}}+\mathrm{b}_{1 \mathrm{k} \cdot \mathrm{g}}
$$

ou g cst la surface terrière de l'arbre en mètres carrés. La figure 4 montre que ce modèle s'adapte bien à chacun des compartiments de l'arbre.

Lcs résultats obtenus font l'objet du tableau 4. La biomasse totale sur pied à 16 ans est voisine de 90 tonnes/ha, dont la moitié constituée par le tronc.

\section{Conclusion}

Sur le plan descriptif nous avons réalisé une investigation poussée au niveau de la structure des houppiers des arbres échantillonnés. Celle-ci conduit à une modélisation de la biomasse des branches : les variables dimensionnelles classiques (D 10 et position dans la couronne traduite ici par l'âge de la branche) donnent un ajustcment très correct aux valeurs mesurées. En outre la comparaison des équations individuelles (établies arbre par arbre) à l'équation générale (établic pour toute la population des branches échantillonnées) montre que le comportement d'un «individu branche »n'est pas indépendant de l'arbre auquel il appartient, bien que cet effet soit difficile à relier aux paramètres mesurés.

Sui le plan des évaluations de la biomasse totale des branches, la modélisation nécessite l'intervention de paramètres différents suivant que l'on s'adresse aux feuilles cu au bois des branches. Ces paramètres sont rarement pris en compte dans les études globales. Il ressort que la modélisation de la biomasse des compartiments d'un arbre doit se faire en utilisant deux types de variables :

a) des variables contemporaines de l'âge du compartiment en question (par exemple la croissance aciuelle de l'arbre pour lexplication de la masse foliaire, ou la circonférence à la base de la cime pour la biomasse ligneuse des branches du houppier) ;

b) des variables de la croissance initiale (quand elles deviennent significatives) pouvant être interprétées comme représentatives d'un caractère intrinsèque de l'arbre vraisemblablement avant son entrée en concurrence avec ses voisins; ce dernier point ouvre des perspectives sur des recherches complémentaires d'intérêt sylvicole.

Une démarche parallèle a été entreprise pour les éléments minéraux. Celle-ci débouchera entre autre sur l'optimisation de l'échantillonnage (choix des échantillons, 
réduction maximum de leur nombre). Pour l'évaluation de la biomasse compartimentée du peuplement nous avons adopté une démarche beaucoup plus classique ; le modèle linéaire retenu est beaucoup plus simple, ceci étant rendu nécessaire par le peu d'information que l'on obticnt par les mesures non destructives.

Reçu le 27 muss 1985.

Accepté le 4 juin 1985.

\section{Remerciements}

Nous tenons à remercier ici $\mathrm{M}^{\mathrm{m} "}$ Ranger, $\mathrm{M}^{\mathrm{II} "}$ Masar ct MM. Adrian, Bouchard, Lefevre, Lefrou, Sartolou, Boireau, Guedon, Lafon† et Rossetto pour les travaux de terrain et de laboratoire, M. Millier pour ses conseils en analyse des données, et M. Darnaudery pour l'exécution des dessins.

\section{Summary \\ Biomass and growth of maritime pine. A study of variability in a 16 years old stand.}

This work is part of a gencral study of the Landes forest ecosystem [one million hectares of Pinus pinctster (Ait.)]. The aim was to determine the economic efficiency of the mineral fertilization used there, but also to evaluate the numerous demands made of this ecosystem (e.g., the effect of whole tree harvesting on production) and their effects on the equilibrium of mineral fertility.

An understanding of the biogeochemical cycle is very important for the poor soils in this region (humic podzols, with a permanent water table). This required an evaluation of reserves (biomass and mineralomass of the stand and of the soil) and also of the principal soil-plant (removals) and plant-soil (returns) fluxes.

We give here the results relating to the morphology and biomass of a young stand (16 years old) on wet heath, which is representative of the Landes sylviculture. An estimate of the biomass was made for each part of the trees in the stand - the needles, branches, wood and bark from the trunk, and roots (excluding the finc roots) - using the most common type of sampling: a stratified sampling related to the inventory of the circumferences of the trees in the plot.

We took advantage of the estimates obtained for 20 trees, to determine whether it would be possible to reduce the sampling in future studies. For that, the maximum relationship was studied between, the biomass of the trees, and the variables, except circumference, which were available or easily collected for all the trees in the plot.

After investigation of the structure of the crown, a model of the biomass of the branches as a function of their diameter (D 10) and age, was obtained. It showed also that part of the variance is due to a tree effect, but the variable responsible could not be integrated into the model; this was duc, among other reasons, to the very small size of the sample.

Regarding the biomass of the crown, the modelling required the inclusion of different parameters, depending on whether it was to be used for the needles or the branch wood. Two types of variables must be taken into account :

i) the variables which are contemporaneous with the age of the part of the tree (for example, the new growth of the trunk explains the mass of foliage, and the circumference of the trunk at the crown base relates to the biomass of the branches). 
ii) The variables of initial growth which represent the intrinsic character of the tree, before the effect of competition.

To determine the biomass of the stand it was necessary to choose simplified models, using only easily measurable parameters but which were still representative of the stand.

Key words: Biomass, growth, modelling, Pinus pinaster (Ait.).

\section{Références bibliographiques}

Baskerville G.L., 1972. Use of logarithmic regression in the estimation of plant biomass. Can. J. For., 2, 49.

BuIs R., 1967. Recherches factorielles sur la régulation de la croissance de l'hypocotyle de Lupin (Lupinus Albus L.). Physiol. Végét., 5 (1), 1-36.

Decourt N., Llimoine B., 1969. Tables de production provisoires pour le pin maritime dans le Sud-Ouest de la France. Amn. Sci. For., 26 (1), 3-14.

Illy G., Lemoine B., 1970. Densité de peuplement, concurrence et coopération chez le pin maritime. I. Premiers résultats d'une plantation à espacement variable. Ann. Sci. For., 27 (2), 127-155.

Kittredge J., 1944. Estimation of the amount of foliage of trees and stands. $J$. For., 42, 905-912.

Kremer A., 1981. Déterminisme génétique de la croissance en hauteur du pin maritime (Pinus pinaster Ait.). I. Rôle du polycyclisme. Ann. Sci. For., 38 (2), 199-222.

Lemoine B., 1980. Densité de peuplement, concurrence et coopération chez le pin maritime. II. Résultats à 5 et 10 ans d'une plantation à espacement variable. Ann. Sci. For., 37 (3), 217-237.

Lemoine B., Gelpe J., Ranger J., Nys C., 1983. Biomasses et minéralomasses du pin maritime. Etude d'un peuplement de 16 ans. In : Annales Congrès I.U.F.R.O. : Mesures des biomasses et accroissements forestiers. Orléans, 3-7 octobre 1983.

MADGWick H.A.I., JACKSON D.S., 1974. Estimating crown weights of Pinus radiata from branch variables. N.Z. J. For. Sci., 4 (3), 520-528.

Ranger D., Nys C., Ranger J., 1981. Etude comparative de deux écosystèmes forestiers feuillu et résineux des Ardennes primaires françaises. II. Biomasse aérienne d'une plantation équienne d'Epicéa commun (Picea abies Kart.). Ann. Sci. For., 38 (3), 377-388.

Satoo T., Madgwick H.A.I., 1982. Forest biomass. Martinus Nijhoff/Dr. W. Junk Publishers, 152 pp. 